Reviews

\title{
Theoretical Simulation of Scanning Probe Microscopy
}

\author{
Masaru TsuKADA \\ WPI Advanced Institute for Materials Research, Tohoku University, Katahira 2-1-1, Aoba, Sendai 980-8577, Japan
}

\begin{abstract}
Methods of theoretical simulation of scanning probe microscopy, including scanning tunneling microscopy (STM), atomic force microscopy(AFM) and Kelvin prove force microscopy (KPFM) have been reviewed with recent topics as case studies. For the case of the STM simulation, the importance of the tip electronic states is emphasized and some advanced formalism is presented based on the non-equilibrium Green's function theory beyond Bardeen's perturbation theory. For the simulation of AFM, we show examples of 3D-force map for AFM in water, and theoretical analyses for a nanomechanical experiment on a protein molecule. An attempt to simulate KPFM images based on the electrostatic multi-pole interaction between a tip and a sample is also introduced.
\end{abstract}

(Received September 29, 2010; Accepted November 12, 2010; Published February 10, 2011)

\begin{tabular}{llll}
1 Introduction & 121 & 6 Simulation Method of Kelvin Probe Force & 124 \\
2 Simulation of STM & 121 & Microscopy & 126 \\
3 Simulation of AFM Images & 122 & 7 Summary and Outlook & 126 \\
4 Theory of SPM in Water & 123 & 8 Acknowledgements & 126 \\
5 SPM Simulation for Protein Molecules & 124 & 9 References & \\
\hline
\end{tabular}

\section{Introduction}

Scanning probe microscopy (SPM) is a family of powerful experimental methods used to observe atomic-scale structures and properties of nano-scale materials. ${ }^{1,2}$ SPM is composed of a variety of methods, such as scanning tunneling microscopy (STM), contact or noncontact atomic force microscopy (AFM), Kelvin probe force microscopy (KPFM) and many others. SPM can observe not only geometrical structures of materials, but also their electronic structures, local mechanical properties, charge distributions, contact potential, local dielectric response, and various other materials properties with atomic resolution. It can also be used to induce atom manipulation and microscopic fabrications. ${ }^{3}$

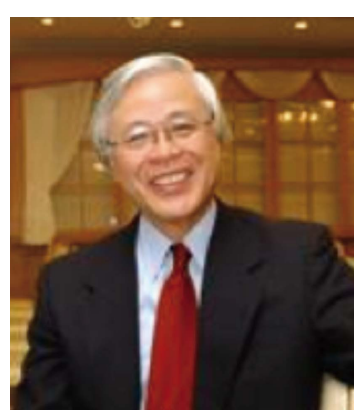

Masaru TsuKada received his D.Sc. Degree from Graduate School of Science, University of Tokyo in 1970, and became a Res. Associate of Dept. Phys., Univ. Tokyo. In 1976, he moved to Inst. Molecular Sci. as an Associate Professor, and returned to Phys. Dept. Univ. Tokyo in 1982, and became Professor at Dept. of Phys., Univ. Tokyo in 1991. In 2004, he became Professor of Waseda Univ. Since 2008, he has been a Professor at WPIAIMR, Tohoku University. His research area includes the theory of surfaces, interfaces, and nano-structures with approaches of computational physics.

E-mail: tsukada@wpi-aimr.tohoku.ac.jp
In spite of very profound information that SPM can in principle provide, it is not easy to analyze detailed experimental data elucidating the true structures and properties of materials. A firm basis for atom or nano-scale manipulation by a theory and theoretical simulations ${ }^{4,5}$ is also necessary. This is because the measured quantities by SPM are obtained by a rather strong or subtle atomic-scale local interaction between the tip and the sample. ${ }^{1,2}$ In the following sections, some topics are introduced based on results obtained by our attempt to develop a versatile theoretical SPM simulator.

\section{Simulation of STM}

When the distance between the tip and the sample is not very small, the tunnel current between them can be well described by Bardeen's perturbation theorem, as below: ${ }^{6}$

$$
I(\mathbf{R}, V)=\frac{2 \pi e}{\hbar} \sum\left\{f\left(E_{\mu}\right)-f\left(E_{v}+e V\right)\right\}\left|M_{\mu v}\right|^{2} \delta\left(E_{\mu}-E_{v}\right),
$$

where $\mathbf{R}$ is the tip position, $V$ the applied bias voltage, $f$ the Fermi distribution function, $E_{\mu}, E_{v}$ the eigen energies of the electronic state at the tip side and sample side, respectively. The tunneling matrix element, $M_{\mu \nu}$, is given by

$$
M_{\mu v}=-\frac{\hbar^{2}}{2 m} \iint_{\mathrm{S}} \mathrm{d} \mathbf{S}\left(\psi_{\mu}^{*} \nabla \psi_{v}-\psi_{v} \nabla \psi_{\mu}^{*}\right)
$$

with a surface integral over an appropriate interface deviding the tip side and the sample side. By using the LCAO scheme, 
which is convenient for most practical calculations, Eq. (1) for the tunnel current is also expressed as ${ }^{4,7}$

$$
I(\mathbf{R}, V)=\frac{2 \pi e}{\hbar} \int_{\mathrm{E}_{\mathrm{F}}}^{\mathrm{E}_{\mathrm{F}}+\mathrm{eV}} \sum_{\mathrm{i}^{\prime}} \sum_{\mathrm{j}^{\prime}} G_{\mathrm{ii}^{\prime}}^{\mathrm{S}}(E) V_{\mathrm{i}^{\mathrm{j}^{\prime}}}(\mathbf{R}) G_{\mathrm{j}^{\prime} \mathrm{j}}^{\mathrm{T}}(E+e V) V_{\mathrm{ji}}(\mathbf{R}) \mathrm{d} E,(3)
$$

where $V_{\mathrm{ij}}$ is the electron hopping integral between sites $\mathrm{i}$ and $\mathrm{j}$. $G_{\mathrm{ij}}^{\mathrm{S} / \mathrm{T}}$ in Eq. (3) is the imaginary part of the Green's function of the tip or surface, given by

$$
G_{\mathrm{ij}}^{\mathrm{S} / \mathrm{T}}(E)=\sum_{v} C_{\mathrm{vi}}^{*} C_{\mathrm{vj}} \delta\left(E-E_{\mathrm{v}}^{\mathrm{S} / \mathrm{T}}\right),
$$

where $E_{v}^{\mathrm{S} / \mathrm{T}}$ and $C_{\mathrm{vj}}$ are the energy and orbital coefficients of the $v$ th eigen state at site $\mathrm{j}$, respectively.

Equations (1) - (3) clearly show that the tunnel current is determined by the overlap of the wave functions at the tip and the sample, and thus the above formulae represent the quantum wave nature of electrons. If the wave functions are spherical symmetric (s-waves) around a certain protruded position of the tip, the tunnel current for the small bias voltage is shown to be in proportion to the local density of states (LDOS) of the sample at that position at the Fermi energy. This simple approximation is often used, but is not correct for general cases, and we must use Eq. (2) or (3) for reliable simulations.

For example, Figs. 1(a) and 1(b) show the calculated map of the LDOS and the tunnel current with the tip model of the $\mathrm{W}_{10}$ [111] cluster (see Fig. 1c) for the free base porphyrin, respectively. Both maps somewhat resemble each other, but are not the same in detail, showing different features.

Though Eq. (3) gives a practical algorithm for numerical calculations, a more accurate expression for the tunnel current is needed for more elaborate systems, or for systems with stronger tip-sample interaction. An example of such cases is the STM using a molecular decorated tip for samples like adsorbed molecules on the substrate. For that purpose, we reformulate the tunnel current based on non-equilibrium Green's function (NEGF) theory ${ }^{8}$ as

$$
I(\mathbf{R}, V)=\frac{2 e}{h} \int\left(f_{\mathrm{T}}-f_{\mathrm{s}}\right) \operatorname{Tr}\left[\bar{g}^{\mathrm{t}} \Gamma_{\mathrm{t}} g^{\mathrm{t}} V g^{\mathrm{s}} \Gamma_{\mathrm{s}} \bar{g}^{\mathrm{s}} V\right] \mathrm{d} E,
$$

where $f_{\mathrm{T}}, f_{\mathrm{S}}$ are the Fermi distribution function at the tip and the sample side, respectively, $g^{\mathrm{t}}, g^{\mathrm{s}}\left(\bar{g}^{\mathrm{t}}, \bar{g}^{\mathrm{s}}\right)$ are the retarded (advanced) Green's function of the molecule decorating the tip and the sample molecule, respectively. The matrices $\Gamma_{\mathrm{t}}\left(\Gamma_{\mathrm{s}}\right)$ are twice the imaginary part of the self-energy matrix due to the interaction with the tip (substrate) for the tip decorating (sample) molecule, and $V$ is the interaction matrix between the two molecules. It is verified that this advanced expression of the tunnel current, Eq. (5), takes a nearly similar form as Eqs. (3) and (4), except the $\delta$-function in Eq. (4) is replaced by energy profile functions as the Lorentzian.

When the tip-sample distance becomes very small, the lowest perturbation with respect to the interaction $V$ is not sufficient, and we should take into account all of the orders of the term. The result is written as

$$
\begin{gathered}
I(\mathbf{R}, V)=\int\left(f_{\mathrm{T}}-f_{\mathrm{S}}\right) \operatorname{Tr}\left[\Gamma_{\mathrm{t}}\left(1-g^{\mathrm{t}} V g^{\mathrm{s}} V\right)^{-1} g^{\mathrm{t}} V g^{\mathrm{s}} \Gamma_{\mathrm{s}} \bar{g}^{\mathrm{s}} V \bar{g}^{\mathrm{t}} \times\right. \\
\left.\left(1-V \bar{g}^{\mathrm{s}} V \bar{g}^{\mathrm{t}}\right)^{-1}\right] \mathrm{d} E .
\end{gathered}
$$

This expression indicates that if the strength of the interaction increases very much, the tunnel current turns to decrease with a further approach of the tip. Such an effect has been actually observed. $^{9}$

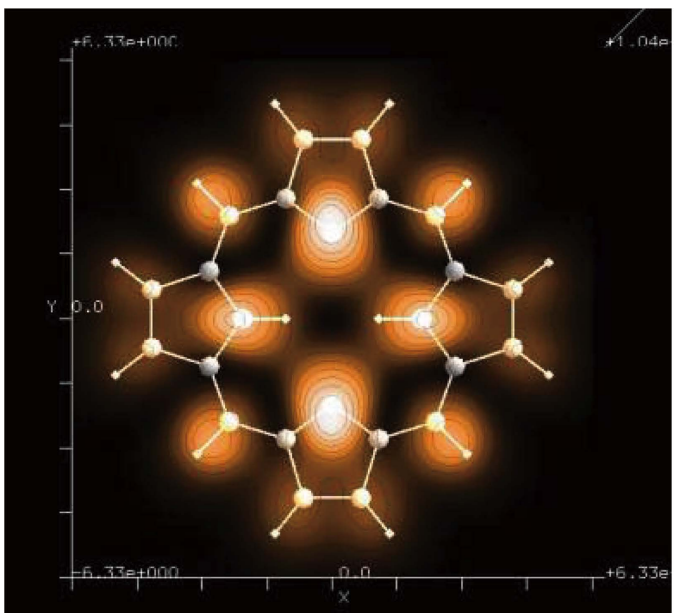

(a)

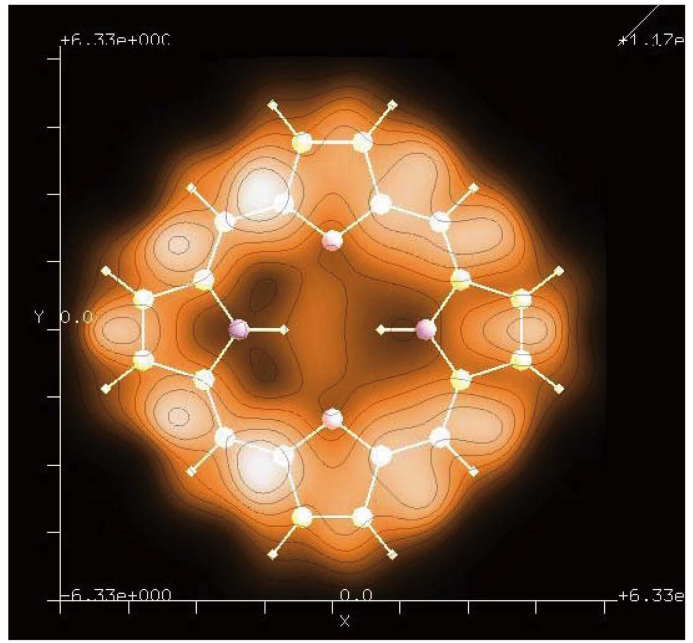

(b)

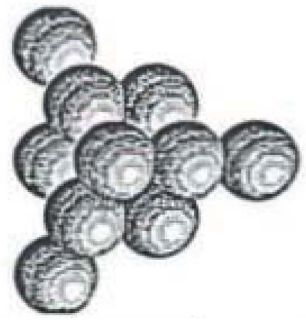

(C)

Fig. 1 (a) Local density of states (LDOS) of porphyrin. (b) STM image of porphyrin by the W[111] tip model including W $6 \mathrm{~s}, 5 \mathrm{~d}$ orbitals. (c) Structure of the tip model, $\mathrm{W}_{10}[111]$, used for the simulation of Fig. 1(b).

\section{Simulation of AFM Images}

For simulating dynamic AFM, the following harmonic oscillator model is often used; ${ }^{10}$

$$
\frac{\mathrm{d}^{2} x}{\mathrm{~d} t^{2}}+\gamma \frac{\mathrm{d} x}{\mathrm{~d} t}+\omega_{0}^{2} x=F_{\text {driv }}(t)+F_{\mathrm{TS}}(x),
$$

where $x$ is the displacement of the oscillator from the equilibrium point, $\gamma$ an effective friction coefficient, $\omega_{0}$ the natural resonance frequency of the oscillator for the case without the tip-surface interaction. $F_{\text {driv }}$ and $F_{\mathrm{TS}}$ are the driving force of the cantilever and the tip-sample interaction force, respectively. The above 
harmonic oscillator model can be derived from the macroscopic continuum model of the cantilever by projecting out its oscillation onto a certain eigen mode with the closest eigen angular frequency, $\omega_{0}$, to the driving angular frequency of $F_{\text {driv }}$.

A sort of perturbation treatment of Eq. (7) reveals that the shift of the resonance frequency is given by ${ }^{11}$

$$
\Delta f=-\frac{1}{4 \pi^{2} \omega_{0} A} \int_{0}^{2 \pi} F_{\mathrm{TS}}(A \cos \theta+L) \cos \theta \mathrm{d} \theta,
$$

and the dissipation energy per cycle is given by

$$
\begin{gathered}
G=\frac{1}{\pi \omega_{0}} \int_{0}^{2 \pi} \gamma(A \cos \theta+L, A \dot{\theta} \sin \theta) \sin ^{2} \theta \mathrm{d} \theta+ \\
\frac{1}{2 \pi^{2} \omega_{0} A} \int_{0}^{2 \pi} F_{\mathrm{TS}}(A \cos \theta+L) \sin \theta \mathrm{d} \theta,
\end{gathered}
$$

where $A$ and $L$ are the amplitude and the height of the equilibrium position of the cantilever, respectively. The frequency shift and the dissipation are used for forming various sorts of dynamic

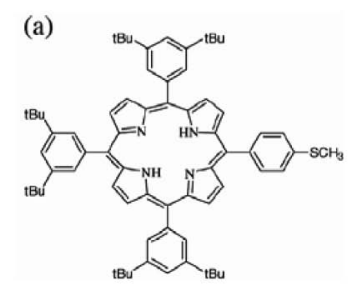

(b)

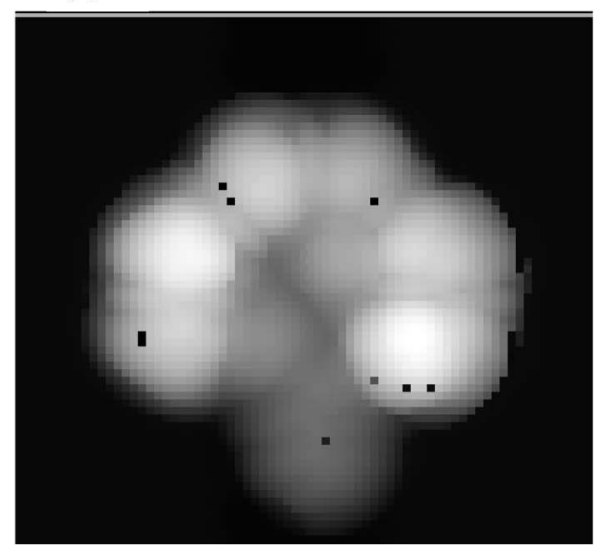

Fig. 2 (a) Structure and (b) simulated AFM image of MSTBPP.
AFM images. The important fact is that once the tip-sample interaction force, $F_{\mathrm{TS}}(z)$, is calculated for each lateral position of the tip, the above quantities are obtained with an appropriate modeling of the friction coefficient, $\gamma(z, \dot{z})$. The second term on the right-hand side of Eq. (9) vanishes for the case of a conservative force. It only appears when the force shows hysteresis, i.e., when different force values appear depending on the approaching or retracting way of the tip. ${ }^{11}$ On the other hand, the first term on the right-hand side of Eq. (9) includes the term due to the thermal fluctuation of the sample and the tip atoms, along with other contributions due to a macroscopic origin. ${ }^{12}$

With the use of a hydrogen atom tip, the AFM image of methylthiophenyl-tris- $t$-buthyl phenyl-porphyrin (MSTBPP) is simulated. ${ }^{13}$ Compared with the experimental image reported in Ref. 14, the theoretical image reproduces the experimental observation fairly well (see Fig. 2)..$^{14}$ In this simulation, the molecular shape and position are fixed. However, if they are allowed to deform due to a strong repulsive force by the tip, regular images are gradually lost, and we observe an interesting deformation behavior of the sample molecule. ${ }^{13}$

Figures 3(a) and 3(b) are the simulated frequency shift image and the dissipation image of the chemisorbed methyl group on a hydrogenated $\mathrm{Si}(100)$ dimer surface, respectively..$^{15}$ The model of the tip used is the $\mathrm{Si}_{4} \mathrm{H}_{9}$ cluster model. The angle of the bond connecting the methyl to the $\mathrm{Si}$ atom is easily bent by a repulsive force by the tip. Therefore, the frequency shift image is rather vague and the hydrogen atom can be observed only as a dark crescent area in Fig. 3(a). The dissipation image shown in Fig. 3(b) produces a rather sharp shape, but the analysis is difficult without a theoretical simulation.

\section{Theory of SPM in Water}

There are several difficult problems for simulating the dynamic AFM in liquids. First, the cantilever bending oscillation as a flexible elastic body should be solved simultaneously with fluid dynamics as shown in Fig. 4. Figure 5 shows a resonance curve of a rectangular silicon slab cantilever in water. ${ }^{16}$ We can see that the resonant frequency is lowered by about $1 / 5$, and the width of the resonance is enlarged by an order of $10^{3-4}$, as compared with those in a vacuum. It is remarkable that the amplitude of the bending angle of the tip apex does not decay very much at the higher frequency side, which reproduces the experimental features.

When a tip approaches a sample, it feels a force mediated by water molecules. This effect to the cantilever motion determines (a)

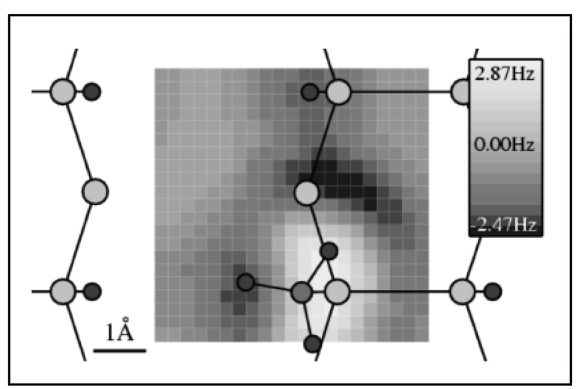

(b)

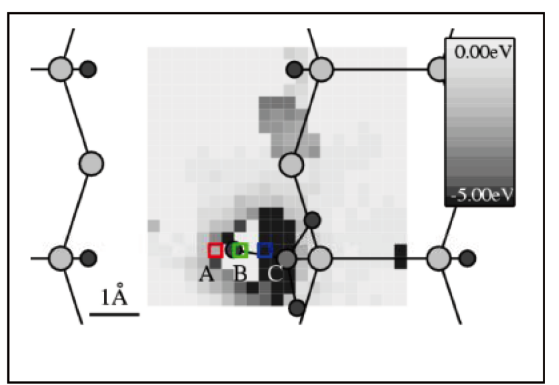

Fig. 3 NCAFM image of the methyl group chemisorbed on the dimer Si atom of a hydrogenated $\mathrm{Si}(100)$ surface taken by the frequency shift (a) and the dissipated energy (b). 


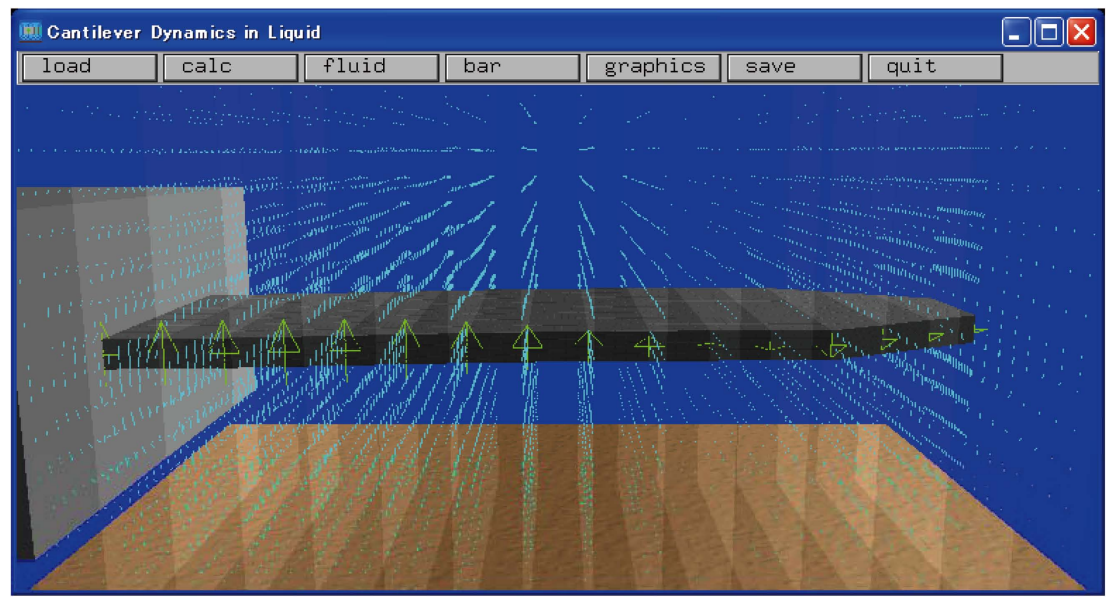

Fig. 4 Oscillation of a cantilever in water.

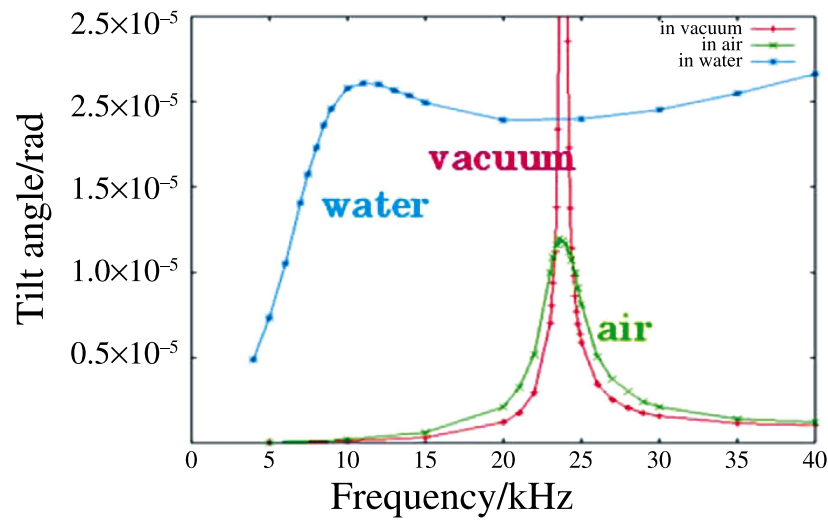

Fig. 5 Resonant curves of a thin Si rectangular cantilever in water, air and in vacuum.

the AFM image in liquids. ${ }^{17,18}$ Figure 6 shows a three-dimensional force map of the CNT (carbon nano-tube) tip over a mica surface in water. It can be seen that an oscillatory solvation force develops near the surface, but the phase of the oscillation sensitively depends on the lateral position of the tip. The oscillatory solvation force is caused by a layer-like distribution of water molecules near the sample and the tip surfaces, as can be seen in Fig. 6(b). These features of the microscopic behavior of the solvated force qualitatively reproduce experiments. ${ }^{19}$

\section{SPM Simulation for Protein Molecules}

Mechanical deformation and stress effect on various physical and chemical properties of protein molecules provide rich information on the functionality of protein molecules, which can be elucidated by theoretical simulations. ${ }^{20}$ Not only AFM images, but also the tip force-distance curve reveals important structural, mechanical, and chemical properties of protein molecules, and thus interpreting these is a major target of the simulation.

As an example, a constant-force AFM image of a single fragment collagen molecule adsorbed on a graphite substrate is calculated both under an ultra-high vacuum and in a wet condition. $^{21}$ As the tip model for this simulation, a narrow capped carbon nano-tube is adopted, as shown in Fig. 7. The effect of the water molecules on the tip-sample interaction force immersing the collagen has also been investigated.

The simulated image of Fig. 8 shows a nodal structure, which reflects the triple-helix structure of the collagen. In the case of the system immersed in water, the amplitude and phase of the oscillatory solvation force are different between on top of the collagen molecule and on top of the graphite surface, which require careful analyses of the image. ${ }^{21}$

Green fluorescent protein (GFP) emits strong green fluorescent light when illuminated with blue light. It has been found that the fluorescent activity of the chromophore, which is located at the hydrophobic cavity inside the $\beta$-barrel structure, is remarkably suppressed on the compression by the tip. ${ }^{22} \mathrm{We}$ performed a simulation of the compression process of the GFP by all-atom molecular mechanics with a hybrid ONIOM9 (QM/QM) calculation as seen in Fig. 9.23 Upon compression, the hydrogen bond network around the chromophore is broken, and the potential barrier for the conformation change disappears, thus opening a non-radiative pathway, which causes quenching of the fluorescence.

\section{Simulation Method of Kelvin Probe Force Microscopy}

In KPFM the electrostatic force between a tip and a sample with an applied voltage $V$ at a fixed tip position $\mathbf{R}$ is conventionally assumed to be

$$
F(\mathbf{R})=-\frac{1}{2} \frac{\partial C(\mathbf{R})}{\partial z}\left(V_{\mathrm{CPD}}-V\right)^{2}
$$

where $z$ is the tip height and $C(\mathbf{R})$ is the capacitance between the tip and the sample, $V_{\mathrm{CPD}}$ being the contact potential difference (CPD), i.e., the difference between the Fermi levels of the tip and the sample.

The above expression indicates that the electrostatic attractive force felt by the tip takes a minimum when the external voltage, $V$ equals to $V_{\text {CPD }}$, which should not depend on the local position of the tip. In actual experiments, however, this value is observed to depend on the microscopic tip position, ${ }^{24-26}$ and is therefore called the local contact potential difference, LCPD. Recently, KPFM images with even atomic resolution have been 

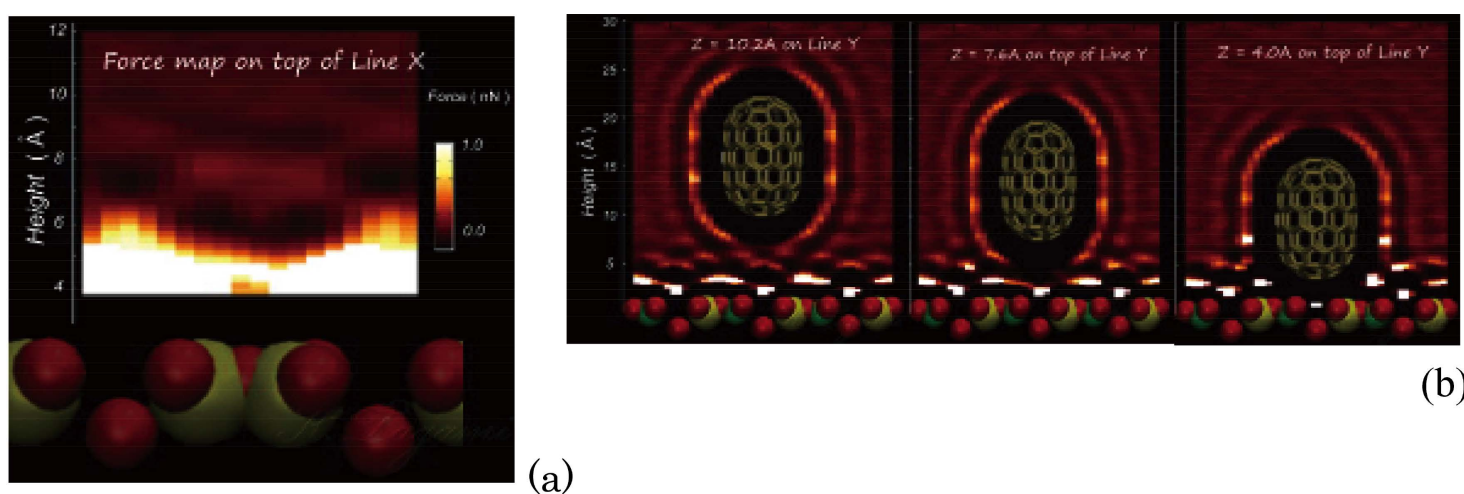

(a)

Fig. 6 (a) 3D force map of mica by a CNT tip and (b) layer structure of the distribution of water molecules near the tip and mica.

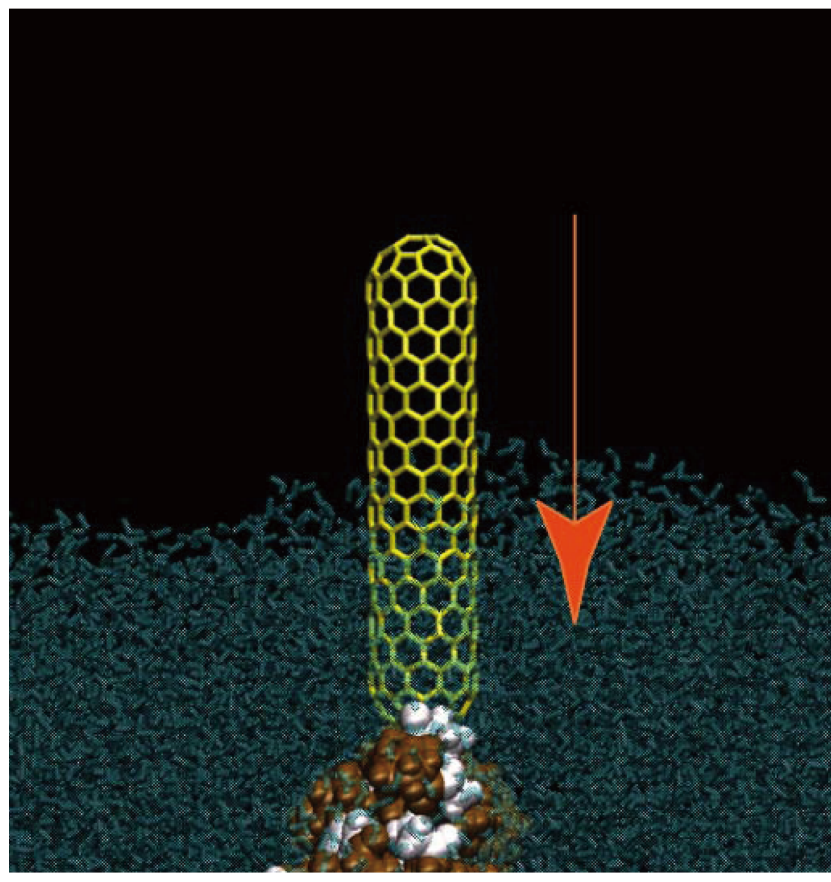

Fig. 7 Simulation model of a CNT tip and a collagen sample immersed in water.

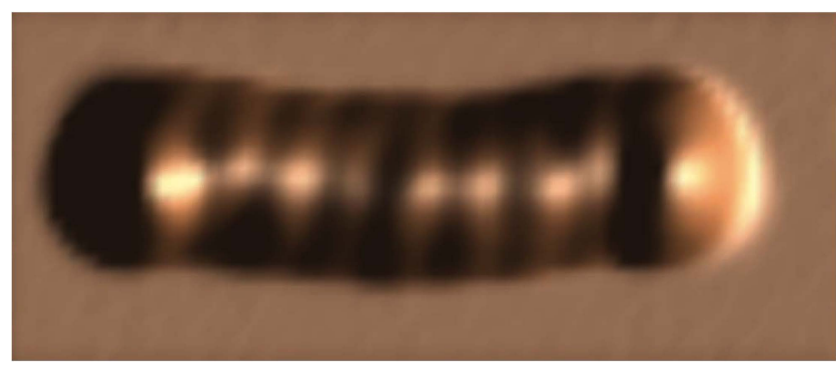

Fig. 8 Simulated constant-force mode AFM of a short collagen model.

reported..$^{27-32}$ This experimental fact is hard to understand based on the model of the electrostatic force described above, and the "local" CPD would be caused by some unknown microscopic effect.
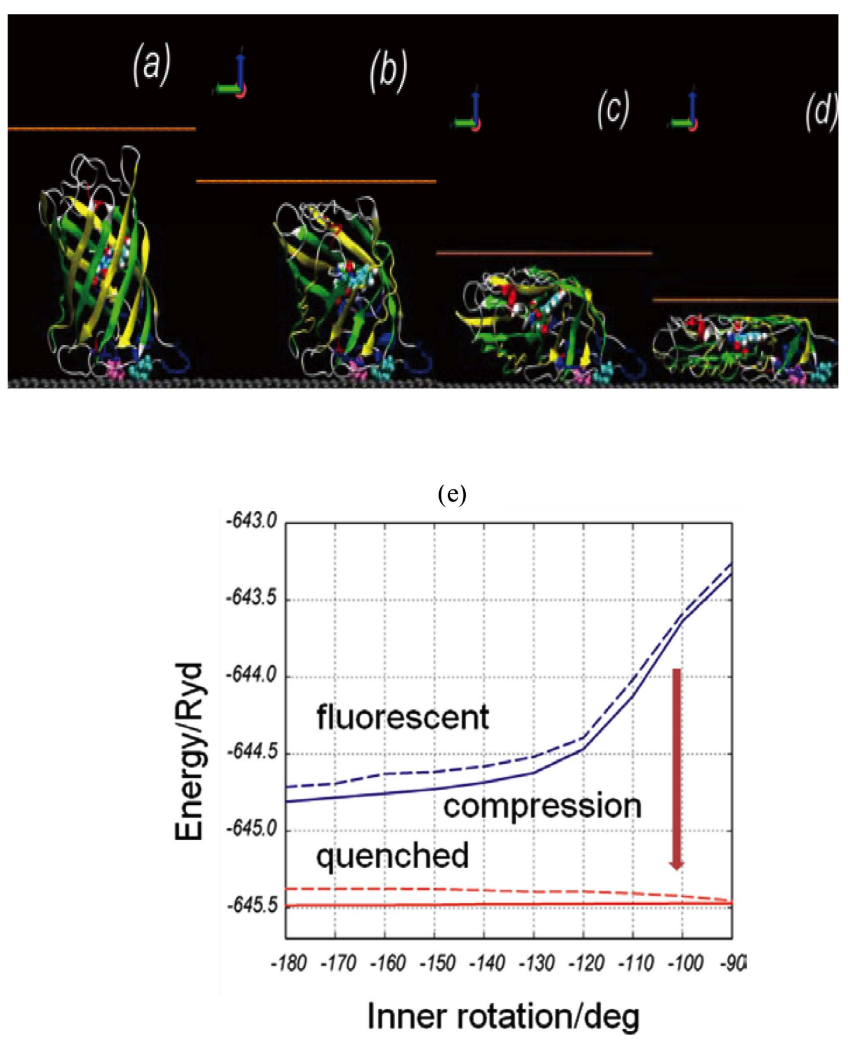

Fig. 9 (a) - (d) Compression of GFP and (e) associated change of the potential energy surface of the excited (dashed line) and ground state (full line).

Toward the elucidation of this puzzle, we have recently developed a general theory of KPFM based on electronic-state calculations using a partitioned real space DFTB (PR-DFTB) method. $^{33}$ This method utilizes the density functional based tight binding (DFTB) method, ${ }^{34}$ and is able to perform self-consistent calculations of a total system composed of two subsystems characterized by different Fermi levels. With the PR-DFTB method, the change of the total energy can be calculated as well as the induced atomistic charge distribution in the tip and the sample by their approach under an applied bias voltage.

Unlike the conventional concept of the Kelvin force, the force acting between the biased tip and the sample depends not only 
on the net charge transferred between the tip and the sample, but also on the multi-pole forces due to the microscopic charge distribution within the sample and the tip. ${ }^{35}$ This causes the mechanism for observing the "apparent" LCPD.

The KPFM images are simulated theoretically by tip models of Si or hydrogenated Si cluster for simple models of clean and hydrogenated $\mathrm{Si}(001)$, $\mathrm{Si}(111)$ surfaces with defects or chemisorbed atoms. ${ }^{35}$

An image of the LCPD for the $\operatorname{Si}(001) \mathrm{c}(2 \times 4)$ surface by the tip models of $\mathrm{Si}_{4} \mathrm{H}_{9}$ is shown in Fig. 10. The tip height is fixed at $0.6 \mathrm{~nm}$ from the surface. In the simulated image the brightest regions in the $V_{\mathrm{LCPD}}$ map correspond to the upper dimer atoms; these regions are connected by the bright zigzag stripe along the dimer row. An important fact is that the above calculated LCPD images show a variation in an atomic scale, the patterns of which are similar as those observed by STM or AFM. ${ }^{36}$

In the simulated LCPD images (not shown here) of the mono-hydride $\mathrm{Si}(001)$ surface with and without a $\mathrm{H}$ vacancy by the tip model of $\mathrm{Si}_{4} \mathrm{H}_{9}$, we have obtained considerably different features. Namely, for the surface without the defect, bright straight stripe patterns appear along the hydrogenated Si dimer row, but the amplitude of the image is considerably small. On the other hand, when a hydrogen atom is removed and a vacancy of the $\mathrm{H}$ adatom is introduced, there appears a very large and bright region centered at the removed $\mathrm{H}$ site; the amplitude of the image is dramatically increased. This is due to the large change of the charge at the dangling bond, i.e., the locally strong polarization at the defect site.

When the tip height is not very small, the individual atoms are not well resolved in KPFM as compared with STM or AFM where the images are taken at lower tip heights. This is because the tip-sample interaction force at the range around $1 \mathrm{~nm}$ is dominantly caused by the electrostatic multi-pole interaction in the case of KPFM. If the tip height becomes smaller, the tip starts to feel a force due to direct orbital hybridization. We have confirmed that in an extended treatment of the PR-DFTB theory to include by a perturbation method, the orbital hybridization effect between the tip and the surface reproduces the atomically resolved KPFM images at the lower tip height region.

\section{Summary and Outlook}

In this article, methods of theoretical simulations of STM, AFM and KPFM have been reviewed with some typical examples obtained in case study research. For most of the cases, the simulations can reproduce experimental images and provide a reliable guide for the interpretation and analyses of experimental data. Even for such difficult situations as AFM measurements in liquids, theoretical simulations can describe the dynamic behavior of liquid molecules intervening the gap between the tip and the sample mediating their interacting force. Theoretical simulations have also been shown successful for giving clear physical insight concerning the nano-mechanical effect on the materials properties of proteins, such as the nano-mechanical quenching of fluorescence of GFP molecules. The theory of KPFM simulation seems to be promising to elucidate the mechanism of microscopic variations of the contact potential difference, and to reveal rich information included in the experimental KPFM data.

\section{Acknowledgements}

This article is based on studies supported by the Japan Science
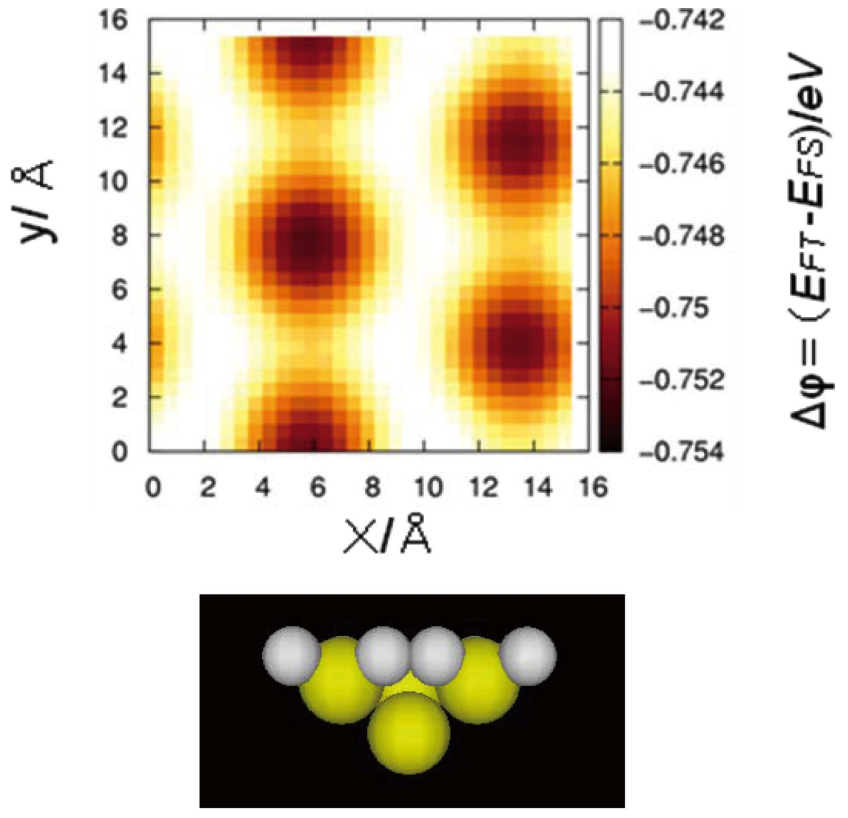

Fig. 10 KPFM image $\mathrm{Si}(001) \mathrm{c}(2 \times 4)$ surface by the model tip of $\mathrm{Si}_{4} \mathrm{H}_{9}$ (bottom).

and Technology Agency program "Development of System and Technology for Advanced Measurement and Analysis". The author would like to give thanks for collaboration with Dr. K. Tagami, M. Harada, A. Masago, N. Watanabe and M. Shimizu.

\section{References}

1. R. Wiesendanger and J. Guentherodt (ed.), "Scanning Tunneling Microscopy I, II, III", 1996, Springer.

2. S. Morita, R. Wiesendanger, and E. Meyer (ed.), "Noncontact Atomic Force Microscopy", 2002, Springer.

3. Y. Sugimoto, M. Abe, S. Hirayama, N. Oyabu, O. Custance, and S. Morita, Nat. Mater., 2005, 4, 156.

4. M. Tsukada, K. Kobayashi, N. Isshiki, and H. Kageshima, Surf. Sci. Rep., 1991, 33, 265.

5. M. Tsukada and S. Hasegawa, "Road Map of Scanning Tunneling Microscopy”, ed. S. Morita, 2006, Springer, $177-1888$

6. J. Bardeen, Phys. Rev. Lett., 1961, 6, 57

7. M. Tsukada and N. Shima, J. Phys. Soc. Jpn., 1987, 56, 2875

8. S. Datta, "Electronic Transport in Mesoscopic Systems", 1995, Cambridge.

9. P. Jelnek, M. Svec, P. Pou, and V. Chab, Phys. Rev. Lett., 2008, 101, 176101.

10. M. Tsukada, N. Sasaki, M. Gauthier, K. Tagami, and S. Watanabe, in "Non-Contact Atomic Force Microscopy", ed. S. Morita, 2002, Springer, $257-278$.

11. N. Sasaki and M. Tsukada, Jpn. J. Appl. Pys., 2000, 59, L1334.

12. M. Gauthier and M. Tsukada, Surf. Sci., 2001, 495, 204.

13. M. Harada and M. Tsukada, Phys. Rev. B, 2008, 77, 205435.

14. S. Tanaka, H. Suzuki, T. Kamikado, and S. Mashiko, Thin Solid Films, 2003, 438, 56

15. A. Masago, S. Watanabe, K. Tagami, and M. Tsukada, $J$. Phys. Conf. Ser., 2007, 61, 1122.

16. M. Tsukada and N. Watanabe, Jpn. J. Appl. Phys., 2009, 
48, 035001-1- 6 .

17. M. Harada and M. Tsukada, Phys. Rev. B, 2010, 80, 035414.

18. M. Tsukada, N. Watanabe, M. Harada, and K. Tagami, J. Vac. Sci., Nanotechnol. B, 2010, B28(3), C4C1.

19. T. Fukuma, Y. Ueda, S. Yoshioka, and H. Asakawa, Phys. Rev. Lett., 2010, 104, 016101.

20. M. Tsukada, K. Tagami, Q. Gao, and N. Watanabe, Current NanoScience, 2007, 3, 57.

21. K. Tagami and M. Tsukada, e-J. Surf. Sci. Nanotechnol., 2006, 4, 294.

22. T. Kodama, H. Ohtani, H. Arakawa, and A. Ikai, Appl. Phys. Lett., 2005, 86, 043901.

23. Q. Gao, K. Tagami, M. Fujihira, and M. Tsukada, Jpn. J. Appl. Phys., 2006, 45, L929.

24. M. Nonnenmacher, M. P. O’Boyle, and H. K. Wickramashinghe, Appl. Phys. Lett., 1991, 58, 2921.

25. T. Glatzel, L. Zimmerli, S. Koch, B. Such, S. Kawai, and E. Meyer, Nanotechnology, 2009, 20, 264016.

26. C.-S. Jiang, H. R. Moutinho, M. J. Romero, M. M. Al-Jassim, and L. L. Kazmerski, Appl. Phys. Lett., 2006, $88,061909$.

27. K. Okamoto, K. Yoshimoto, Y. Sugawara, and S. Morita,
Appl. Surf, Sci., 2003, 210, 128.

28. S. Kitamura, K. Suzuki, M. Iwatsuki, and C. B. Mooney, Appl. Surf. Sci., 2000, 157, 222.

29. F. Bocquet, L. Nony, and C. Loppacher, Phys. Rev. B, 2008 $78,035410$.

30. L. Nony, A. S. Foster, F. Bocquet, and C. Loppacher, Phys. Rev. Lett., 2009, 103, 036802.

31. S. Sadewasser, P. Jelinck, C.-K. Fang, O. Custance, Y. Yamada, Y. Sugitomo, M. Abe, and S. Morita, Phys. Rev. Lett., 2009, 103, 266103.

32. L. Nony, F. Bouquet, C. Loppacher, and T. Glatzel, Nanotechnology, 2009, 20, 264014.

33. N. Nagaoka, K. Tada, S. Watanabe, H. Fujita, and K. Watanabe, Phys. Rev. Lett., 2001, 86, 540.

34. M. Elsterner, D. Porezag, G. Jungnickel, J. Elsner, M. Haugk, Th. Frauenheim, S. Suhai, and G. Seifert, Phys. Rev. B, 1998, 58,7260 .

35. A. Masago, M. Tsukada, and M. Shimizu, Phys. Rev. B, submitted.

36. A. Masago, S. Watanabe, K. Tagami, and M. Tsukada, Jpn. J. Appl. Phys., 2009, 48, 025506 